\title{
DIFFERENTIABLE PROJECTIONS AND DIFFERENTIABLE SEMIGROUPS
}

\author{
J. P. HOLMES
}

\begin{abstract}
Suppose $X$ is a Banach space, $G$ is a connected open subset of $X$, and $p$ is a continuously Fréchet differentiable function from $G$ into $G$ satisfying $p(p(x))=p(x)$ for each $x$ in $G$. We prove that $p(G)$ is a differentiable submanifold of $X$ and use this result to show that the maximal subgroup containing an idempotent in a differentiable semigroup is a Lie group.
\end{abstract}

Suppose $X$ is a Banach space, $G$ is a connected open subset of $X$, and $p$ is a continuously Fréchet differentiable function from $G$ into $G$ satisfying $p(p(x))=p(x)$ for each $x$ in $G$. We prove that $p(G)$ is a differentiable submanifold of $X$ and use this result to show that the maximal subgroup containing an idempotent in a differentiable semigroup is a Lie group.

In [4] Nadler proved that the image of a differentiable projection defined on an open subset of a Euclidean space is a differentiable manifold.

THEOREM 1. Suppose each of $X, p$, and $G$ is as above. There is a closed linear subspace $Y$ of $X$ and a set $H=\left\{h_{x}\right\}$ of functions, one for each $x$ in $p(G)$ so that, for each $x$ in $p(G), h_{x}$ is a homeomorphism from a neighborhood of $x$ in $p(G)$ onto a neighborhood of 0 in $Y, h_{x}(x)=0$, and if each of $x$ and $y$ is in $p(G)$ with $y$ in $\operatorname{dom}\left(h_{x}\right)$ then there is a neighborhood $U_{x y}$ of 0 in $Y$ so that $h_{x} \circ h_{y}^{-1}$ is continuously differentiable on $U_{x y}$.

First, we establish some notation. Since $p^{2}=p$ we have, by the chain rule, that $p^{\prime}(p(x)) \circ p^{\prime}(x)=p^{\prime}(x)$ for each $x$ in $G$. In particular, if $x$ is in $p(G)$ then $p^{\prime}(x)$ is a continuous linear idempotent mapping from $X$ into $X$. Denote by $Y_{x}$ the image of $p^{\prime}(x)$ if $x$ is in $p(G)$. Let $p_{x}$ denote the function defined from $G-x$ to $G-x$ by $p_{x}(y)=p(y+x)-x$ for each $x$ in $p(G)$. Finally, if $d$ is a positive number denote by $R(d)$ the subset of $X$ to which $x$ belongs if and only if $\|x\|<d$.

LEMma 1. If $x$ is in $p(G)$ then there is a $d_{x}>0$ so that $p^{\prime}(x)$ is one-to-one on $R\left(d_{x}\right) \cap p_{x}(G-x)$.

Received by the editors January 18, 1973 and, in revised form, February 26, 1973. AMS (MOS) subject classifications (1970). Primary 52B99; Secondary 22E65.

Key words and phrases. Differentiable manifold, differentiable semigroup. 
Proof. Choose $d_{x}$ by continuity of $p_{x}^{\prime}$ so that if $y$ is in $R\left(d_{x}\right)$ then $y$ is in $G-x$ and $\left\|p_{x}^{\prime}(y)-p_{x}^{\prime}(0)\right\|<\frac{1}{2}$.

Suppose that each of $y$ and $z$ is in $R\left(d_{x}\right) \cap p_{x}(G-x)$.

$$
\begin{aligned}
\|y-z\| & =\left\|p_{x}(y)-p_{x}(z)\right\| \\
& \leqq\left\|p_{x}(y)-p_{x}(z)-p_{x}^{\prime}(0)(y-z)\right\|+\left\|p_{x}^{\prime}(0)(y-z)\right\| \\
& =\left\|\int_{0}^{1} d t\left[p_{x}^{\prime}(z+t(y-z))-p_{x}^{\prime}(0)\right](y-z)\right\|+\left\|p_{x}^{\prime}(0)(y-z)\right\| \\
& \leqq \frac{1}{2}\|y-z\|+\left\|p_{x}^{\prime}(0)(y-z)\right\| .
\end{aligned}
$$

Thus $\frac{1}{2}\|y-z\| \leqq\left\|p_{x}^{\prime}(0)(y-z)\right\|$ and we are done.

Lemma 2. Suppose $x$ is in $p(G)$. There are neighborhoods $U_{x}$ and $V_{x}$ of 0 in $Y_{x}$ so that $f_{x}=p^{\prime}(x) \circ p_{x}$ satisfies:

(a) $\left(f_{x} \mid U_{x}\right)$ is a homeomorphism onto $V_{x}$.

(b) Each of $U_{x}$ and $p_{x}\left(U_{x}\right)$ is contained in $R\left(d_{x}\right)$.

(c) $\left(f_{x} \mid U_{x}\right)^{-1}$ is continuously differentiable on $V_{x}$.

Proof. This is a simple application of the inverse function theorem [2, p. 268] and the observation that $\left(f_{x} \mid Y_{x} \cap(G-x)\right)^{\prime}(0)$ is the identity function on $Y_{x}$.

LemMa 3. If $x$ is in $p(G)$ then $\left(p_{x} \mid U_{x}\right)$ is a homeomorphism onto a neighborhood of 0 in $p_{x}(G-x)$.

Proof. By Lemma 2, $p_{x}$ is one-to-one on $U_{x}$. Choose $e$ positive and less than $d_{x}$ so that $p^{\prime}(x)(R(e))$ is contained in $V_{x}$. If $y$ is in $R(e) \cap p_{x}(G-x)$ then, by Lemma 2, there is a $z$ in $p_{x}\left(U_{x}\right)$ so that $p^{\prime}(x)(z)=p^{\prime}(x)(y)$. Since $p_{x}\left(U_{x}\right)$ is contained in $R\left(d_{x}\right)$ and $e<d_{x}$ we have $y=z$ is in $p_{x}\left(U_{x}\right)$. Hence $p_{x}\left(U_{x}\right)$ is a neighborhood of 0 in $p_{x}(G-x)$.

Let $g_{x}=\left(p_{x} \mid U_{x}\right)^{-1}$ and suppose each of $y$ and $z$ is in $B_{x}=\operatorname{dom}\left(g_{x}\right)$.

$$
\begin{aligned}
\| g_{x}(y) & -g_{x}(z)\|-\| y-z \| \\
& \leqq\left\|g_{x}(y)-g_{x}(z)-(y-z)\right\| \\
& =\left\|p_{x}\left(g_{x}(y)\right)-p_{x}\left(g_{x}(z)\right)-p^{\prime}(x)\left(g_{x}(y)-g_{x}(z)\right)\right\| \\
& =\left\|\int_{0}^{1} d t\left[p_{x}^{\prime}\left(g_{x}(z)+t\left(g_{x}(y)-g_{x}(z)\right)\right)-p_{x}^{\prime}(0)\right]\left(g_{x}(y)-g_{x}(z)\right)\right\| \\
& \leqq \frac{1}{2}\left\|g_{x}(y)-g_{x}(z)\right\|
\end{aligned}
$$

since each of $g_{x}(y)$ and $g_{x}(z)$ is in $R\left(d_{x}\right)$. Thus $\left\|g_{x}(y)-g_{x}(z)\right\| \leqq 2\|y-z\|$ and we are done.

For each $x$ in $p(G)$ let $C_{x}=B_{x}+x$ and define $m_{x}$ on $C_{x}$ by $m_{x}(y)=g_{x}(y-x)$. $C_{x}$ is a neighborhood of $x$ in $p(G)$ and $m_{x}$ is a homeomorphism from $C_{x}$ onto $U_{x}$. 
Lemma 4. Suppose each of $x$ and $y$ is in $p(G)$ and $y$ is in $C_{x}$. Then there is a $d(x, y)>0$ such that $\left(m_{x} \circ m_{y}^{-1} \mid R(d(x, y)) \cap U_{y}\right)$ is continuously differentiable.

Proof. If $y$ is in $C_{x}$ then $y-x$ is in $B_{x} \cdot p_{x}(y-x)=y-x$ so by continuity of $p_{x}$ and + there is a $d(x, y)>0$ so that each of $R(d(x, y))$ and $y-x+$ $R(d(x, y))$ is contained in $G-x$ and $p_{x}(y-x+R(d(x, y)))$ is contained in $B_{x}$.

From Lemma $2,\left(f_{x} \mid U_{x}\right)^{-1}$ is continuously differentiable on $V_{x} . f_{x}$ is continuously differentiable on $G-x$, so the function $n_{x}$ defined on $R(d(x, y))$ by $n_{x}(w)=f_{x}(w+y-x)$ is continuously differentiable.

If $w$ is in $R(d(x, y)) \cap U_{y}$ then

$$
\begin{aligned}
m_{x}\left(m_{y}^{-1}(w)\right) & =m_{x}\left(g_{y}^{-1}(w)+y\right)=m_{x}\left(p_{y}(w)+y\right) \\
& =g_{x}(p(w+y)-x)=\left(p_{x} \mid U_{x}\right)^{-1}\left(p_{x}(w+y-x)\right) \\
& =\left[\left(p_{x} \mid U_{x}\right)^{-1} \circ\left(p^{\prime}(x) \mid B_{x}\right)^{-1} \circ p^{\prime}(x) \circ p_{x}\right](w+y-x) \\
& =\left(f_{x} \mid U_{x}\right)^{-1} \circ\left(n_{x} \mid U_{y} \cap R(d(x, y))\right)(w) .
\end{aligned}
$$

This is the composition of differentiable functions so we are done.

Lemma 5. If each of $x$ and $y$ is in $p(G)$ then $Y_{x}$ is linearly homeomorphic to $Y_{\nu}$.

Proof. First suppose each of $A$ and $B$ is a continuous linear idempotent mapping from $X$ into $X$ and $\|A-B\|<1$. If $I$ denotes the identity mapping on $X$ then $I+A-B$ is invertible and hence $X=(I+A-B)(X)=$ $(I-B)(X)+A(X)$. Thus $B(X)=B(I-B)(X)+B(A(X))=B(A(X))$ and $(B \mid A(X))$ is onto $B(X)$.

Suppose $x$ is in $A(X) .\|x\|-\|B(x)\| \leqq\|A(x)-B(x)\| \leqq\|A-B\|\|x\|$ so $\|B(x)\| \geqq(1-\|A-B\|)\|x\|$. Thus $(B \mid A(X))$ is one-to-one onto $B(X)$ and, by the closed graph theorem, is a linear homeomorphism.

For each $z$ in $p(G)$ choose $D_{z}$ to be a neighborhood of $z$ in $p(G)$ so that if $w$ is in $D_{z}$ then $\left\|p^{\prime}(w)-p^{\prime}(z)\right\|<\frac{1}{2}$.

Let $A_{1}=D_{r}$ and choose inductively $A_{n}=\bigcup\left\{D_{n}: D_{n}\right.$ intersects $\left.A_{n-1}\right\}$ for $n=2,3, \cdots$.

Let $A_{s}=\bigcup_{1}^{x} A_{i} . A_{\text {s }}$ is open in $p(G)$. Suppose $\left\{z_{n}\right\}$ is a sequence in $A_{x}$ and $\left\{z_{n}\right\}$ converges to $z$. Applying $p$ to the sequence and its limit we have: $p(z)=\lim p\left(z_{n}\right)=\lim z_{n}=z$. Hence $z$ is in $p(G)$. (This argument shows $p(G)$ is closed.) There is an $n$ such that $z_{n}$ is in $D_{z} . z_{n}$ is in $A_{m}$ for some $m$ so $z$ is in $A_{m, 1}$. Thus $A_{r}$ is closed in $p(G)$ and hence is $p(G)$.

$y$ is in $A_{n}$ for some $n$ and hence $Y_{y}$ is linearly homeomorphic to $Y_{x}$.

Choose $z$ in $p(G)$ and for each $y$ in $p(G)$ let $T_{\text {" }}$ be a linear homeomorphism from $Y_{u}$ onto $Y_{z}$. 
Let $Y=Y_{z}$ and $h_{x}=T_{x} \circ m_{x}$ for each $x$ in $p(G)$. Since $p^{\prime}(z)$ is an idempotent, $Y_{z}$ is the kernel of $I-p^{\prime}(z)$ and is therefore closed. Moreover, the kernel of $p^{\prime}(z)$ is a closed complement to $Y_{z}$ in $X$, so we satisfy the conditions for a submanifold to be modeled on $Y$. The closed subspace $Y$ and set of homeomorphisms $\left\{h_{x}: x\right.$ is in $\left.p(G)\right\}$ satisfy the conclusion of Theorem 1.

The next theorem uses Theorem 1 to obtain some information on idempotents in differentiable semigroups.

Theorem 2. Suppose $X$ is a Banach space, $D$ is an open set of $X$ containing 0 , and $V$ is a continuously differentiable associative function from $D \times D$ into $X$ satisfying $V(0,0)=0$. Then the set of elements $x$ of $D$ satisfying $V(x, 0)=V(0, x)=x$ is locally topologically isomorphic to a local Lie group.

Proof. Define $p$ contained in $D \times X$ by $p=\{(x, V(0, V(x, 0))): V(x, 0)$ is in $D\} . p$ is continuously differentiable on a neighborhood of 0 and $p(p(x))=p(x)$ if $p(x)$ is in $D$.

As before, $p^{\prime}(0)$ is an idempotent continuous linear transformation from $X$ into $X$. Let $Y=p^{\prime}(0)(X)$. From the proofs of Lemmas 2 and 3 there are open sets $U$ and $V$ of $Y$ containing 0 and an open set $E$ of the image of $p$ containing 0 such that $\left(p^{\prime}(0) \circ(p \mid U)\right)^{-1}$ is a continuously differentiable homeomorphism from $V$ onto $U$ and $(p \mid U)$ is a homeomorphism onto $E$.

Let $g=(p \mid U)^{-1}$ and let $A=\left\{(x, y)\right.$ in $U \times U: V\left(g^{-1}(x), g^{-1}(y)\right)$ is in $\operatorname{dom}(g)\}$. $A$ is open in $Y \times Y$. Define $W$ on $A$ by $W(x, y)=g\left(V\left(g^{-1}(x)\right.\right.$, $\left.\left.g^{-1}(y)\right)\right)$.

$$
\begin{aligned}
W(x, y) & =\left[(p \mid U)^{-1} \circ\left(p^{\prime}(0) \mid E\right)^{-1}\right] \circ p^{\prime}(0) \circ V \circ(p \times p)(x, y) \\
& =\left(p^{\prime}(0) \circ(p \mid U)\right)^{-1} \circ p^{\prime}(0) \circ V \circ(p \times p)(x, y)
\end{aligned}
$$

if $(x, y)$ is in $A$. Thus $W$ is continuously differentiable on a neighborhood of $(0,0)$ in $Y \times Y$ and $W(x, 0)=x=W(0, x)$ for each appropriate $x$ in $Y$. Hence, from [1] or in another way from [3], $(Y, W, 0)$ is a local Lie group. $g^{-1}$ is the topological isomorphism required for the conclusion of Theorem 2.

\section{REFERENCES}

1. G. Birkhoff, Analytical groups, Trans. Amer. Math. Soc. 43 (1938), 61-101.

2. J. Dieudonné, Fondements de l'analyse moderne, Pure and Appl. Math., vol. 10, Academic Press, New York and London, 1960. MR 22 \#11074.

3. J. P. Holmes, Differentiable power associative groupoids, Pacific J. Math. 41 (1972), 319-394.

4. S. B. Nadler, Jr., A characterization of the differentiable submanifolds of $R^{n}$, Proc. Amer. Math. Soc. 17 (1966), 1350-1352. MR 34 \#3586.

Department of Mathematics, Auburn University, Auburn, Alabama 36830 